\title{
OPEN SURFACES WITH CONGRUENT GEODESICS
}

\author{
by CHARLAMBOS CHARITOS
}

(Received 27th October 1993)

The aim of this paper is to prove the Theorem: Let $M$ be a complete non compact surface without boundary
in the euclidean space $\mathbb{E}^{3}$. We suppose that all geodesics of $M$ are congruent. Then $M$ is an affine plane in $\mathbb{E}^{3}$.

1991 Mathematics subject classification: 53A05.

If $M$ is a closed surface in the euclidean 3-space which has all its geodesics congruent, then $M$ is a round sphere. Compactness of $M$, which implies that $M$ is a diffeomorphic to a sphere, is crucial in establishing the above result (see [3]).

Similarly, in the study of manifolds with families of congruent curves, compactness is an essential hypothesis (see $[2,6,10]$ ).

In the present note following the principal ideas of [3] we are able, for the first time, to remove the compactness assumption. In fact we show:

Theorem. Let $M$ be a complete non-compact surface without boundary embedded in the euclidean space $\mathbb{E}^{3}$. We suppose that all geodesics of $M$ are congruent. Then $M$ is an affine plane in $\mathbb{E}^{3}$.

In the course of the proof we will often refer to the compact case [3]. However, we will make this paper as self-contained as possible by introducing all necessary notation and definitions.

Proof of the theorem. We separate the proof in several lemmas.

Lemma 1. The surface $M$ is diffeomorphic to $\mathbb{R}^{2}$.

Proof. At first we show that all congruent geodesics of $M$ are simple curves diffeomorphic to $\mathbb{R}$.

Suppose that the geodesics of $M$ have self-intersection points. We pick such a geodesic $\gamma$. In the following we suppose that all the parametrizations of the geodesics or of the geodesic arcs that we consider are by arc-length. Let $f:(-\infty, \infty) \rightarrow M$ be a parametrization of $\gamma$ with $f(0)=p$ and let $\rho>0$ such that $f /[0, \rho]$ has at least one selfintersection point. Since $M$ is an open manifold it is well known that there exists a geodesic ray $r:[0,+\infty) \rightarrow M$ with $r(0)=p$. Therefore $r /[0, \rho]$ is an embedding in $M$ and 
consequently $r^{\prime}(0) \neq f^{\prime}(0)$. We fix an orthonormal basis $\left\{e_{1}, e_{2}\right\}$ of $T_{p} M$ with $e_{1}=r^{\prime}(0)$, which induces an orientation on $T_{p} M$. We parametrize each unit vector $v$ of $T_{p} M$ by the oriented angle $\theta=\Varangle\left(v, e_{1}\right), 0 \leqq \theta<2 \pi$; note that $\Varangle\left(e_{1}, e_{2}\right)=\pi / 2$.

Let now $\theta_{0}=\sup \left\{\theta \in[0,2 \pi]\right.$ such that every geodesic arc $g:[0, \rho] \rightarrow M$ with $g^{\prime}(0) \in T_{p} M$ and $\Varangle\left(g_{0}^{\prime}(0), e_{1}\right)=\theta^{\prime}<\theta$ is simple $\}$. Note that $\theta_{0}>0$ since the set of embeddings $g$. $[0 . \rho] \rightarrow M$ is open in the space $C^{\infty}([0, \rho], M)[5]$. Now we consider the geodesic $g_{0}$ : $[0, \rho] \rightarrow M$ with $g_{0}^{\prime}(0) \in T_{p} M$ and let $\Varangle\left(g_{0}^{\prime}(0), e_{1}\right)=\theta_{0}$. Claim: $g_{0} /[0, \rho]$ is a simple geodesic arc. From this we conclude that $\theta_{0}=2 \pi$ which contradicts the hypothesis that $f /[0, \rho]$ has self intersection points. To prove the claim observe that if $g_{0} /[0, \rho]$ were not simple then every geodesic arc $g:[0, \rho] \rightarrow M \varepsilon$-close to $g_{0} /[0, \rho]$, for $\varepsilon$ small enough, would not be simple. But this contradicts the definition of $\theta_{0}$.

Suppose now that $\pi_{1}(M) \neq 1$. It is well known (see for example [4, Ch. 10, Th. 13] that for every pair of points $p, q$ (and hence for $p=q$ ) and for every arc $\alpha(p, q)$ joining $p, q$ there is a geodesic arc $\gamma(p, q)$ in the homotopy class of $\alpha(p, q)$ with end points fixed. So if we take a noncontractible loop $\alpha(p, p)$ on $M$ and if we consider a geodesic arc $\gamma(p, p)$ in the homotopy class of $\alpha(p, p)$ with $p$ fixed, then the geodesic of $M$ containing $\gamma(p, p)$ is either closed or it has self-intersection points. But this is impossible since we have proved that all geodesics of $M$ are curves diffeomorphic to $\mathbb{R}$. Therefore $\pi_{1}(M)=1$ and $M$ is diffeomorphic to $\mathbb{R}^{2}$.

Now we consider a fixed curve $\Gamma_{0}$ in $\mathbb{E}^{3}$ such that every geodesic of $M$ is congruent to $\Gamma_{0}$. If $\Gamma_{0}$ is a plane curve or if the curvature of $\Gamma_{0}$ is constant then in each case we can easily deduce that all points of $M$ are umbilical and consequently $M$ is an affine plane in $\mathbb{E}^{3}$. We next assume that $\Gamma_{0}$ is not a plane curve as well as that the curvature of $\Gamma_{0}$ is not constant and we will prove that this assumption is incompatible with the hypothesis that all geodesics of $M$ are congruent. Let $\alpha(s), s \in(-\infty, \infty)$ be a parametrization by arc length of $\Gamma_{0}$ and let $k(s), \tau(s)$ be the curvature and torsion functions of $\alpha(s)$ respectively.

We denote by $<,>$ the usual inner product in $\mathbb{E}^{3}$ and by $A$ the shape operator of $M$. Let $v_{p}$ be a vector in the unit tangent bundle $S(M)$ of $M$. There exists a unique geodesic $\gamma:(-\infty, \infty) \rightarrow M$ such that $\gamma(0)=p, \gamma^{\prime}(0)=v_{p}$. We denote by $\kappa\left(v_{p}\right), \tau\left(v_{p}\right)$ the normal curvature and torsion of $\gamma$ at $p$, and we have that:

$$
\kappa\left(v_{p}\right)=\left\langle A v_{p}, v_{p}\right\rangle, \tau\left(v_{p}\right)=\left\langle A v_{p}, J v_{p}\right\rangle,
$$

where by $J v_{p}$ we denote the vector that we obtain if we rotate $v_{p}$ counterclockwise in $T_{p} M$ by $\pi / 2$.

In what follows we will refer to them as the curvature and torsion of vectors of $S(M)$.

Lemma 2. (a) Let $r: S(M) \rightarrow \mathbb{R}^{+}$be the differentiable function defined by $r\left(v_{p}\right)=$ $\left|\kappa\left(v_{p}\right)\right|$ and let $k_{0}$ be a non-critical value of $k(s)$. Then the set $r^{-1}\left(k_{0}\right)$ is a closed surface in $S(M)$.

(b) We can choose the non-critical value $k_{0}$ such that there exists a component $C$ of $r^{-1}\left(k_{0}\right)$ which contains only non-principal vectors. Moreover, for each $v_{p}$ in $C, \tau\left(v_{p}\right)=$ constant $\neq 0$. 
Proof. For the proof of (a) we remark that if $k_{0}$ is a non-critical value of $k(s)$ then $r$ is of rank 1 on $r^{-1}\left(k_{0}\right)$ (for more details see the proof of Proposition 2 in [3]).

For the proof of (b) we consider a non-umbilical point $q$ in $M$; remark that such a point exists since the function $k(s)$ is not constant. Now we can choose a non-principal vector $w_{q}$ in $T_{q} M$ such that $r\left(w_{q}\right)=k_{0}$ and $k_{0}$ is a non-critical value of $k(s)$. Among the components of the surface $r^{-1}\left(k_{0}\right)$ consider that one which contains the vector $w_{q}$ and denote it by $C$. We can prove that $\tau\left(v_{p}\right)=$ constant $\neq 0$ for each $v_{p}$ in $C$ which implies that all the vectors of $C$ are non-principal (for more details see the proof of the lemma in [3]).

Lemma 3. Let $\pi: C \rightarrow M$ be the projection in $M$ with $\pi\left(v_{p}\right)=p$. Then the pair $(C, \pi)$ is $a$ covering space of $M$.

Proof. As in Proposition 3 of [3] we prove that $\pi$ has rank 2 at every $v_{p}$ in $C$, so $\pi$ is a local diffeomorphism. We next show that $\pi$ is onto by proving that $\pi(C)$ is an open and closed subset in $M$. Since $\pi$ is a local diffeomorphism we get that $\pi(C)$ is an open subset of $M$ and next we will prove that $\pi(C)=\overline{\pi(C)}$ which implies that $\pi(C)$ is also closed in $M$. Let $p \in \overline{\pi(C)}$, then there is a sequence $p_{n}$ in $\pi(C)$ which converges to $p$. Let $v_{n} \in C$ with $\pi\left(v_{n}\right)=p_{n}$. Since $M$ is diffeomorphic to $\mathbb{R}^{2}$ we have that $S(M)$ is diffeomorphic to $M \times S^{1}$ under a diffeomorphism $F$. Let $\left(p_{n}, \theta_{n}\right)=F\left(v_{n}\right)$. The space $S^{1}$ is compact so there exists a subsequence $\theta_{n_{\kappa}}$ of $\theta_{n}$ converging to a $\theta \in S^{1}$. Consequently $\left(p_{n_{\kappa}}, \theta_{n_{k}}\right)$ converges to $(p, \theta)$; hence the subsequence $v_{n_{k}}$ of $v_{n}$ converges to a $v$ in $C$ since $C$ is a closed subset in $S(M)$. It follows that $p=\pi(v)=\lim _{\kappa} \pi\left(v_{n_{\kappa}}\right)$ belongs to $\pi(C)$ which implies that $\pi(C)=\overline{\pi(C)}$.

Observe that $M$ is simply connected and therefore has no non-trivial covering spaces. So the projection $\pi$ : $C \rightarrow M$ is a diffeomorphism. This permits the construction of a nonvanishing vector field $X$ on $M$ such that $r\left(X_{p}\right)=$ constant for each $p$ in $M$.

Lemma 4. The set of non-critical values $k_{0}$ of the curvature function $k(s)$, such that some component of $r^{-1}\left(k_{0}\right)$ contains non-principal vectors, is dense into the range $R$ of $k(s)$.

Proof. At first we know by Sard's theorem [5] that the set of non-critical values of $k(s)$ is dense in $R$. Let $k_{0}$ be a non-critical value of $k(s)$ such that $r^{-1}\left(k_{0}\right)$ contains only principal vectors. Let $v_{p}$ be such a vector in $r^{-1}\left(k_{0}\right)$. We distinguish 2 cases:

(1) The point $p$ is non-umbilical. Suppose without loss of generality that $k_{0}=r\left(v_{p}\right)$ is the minimum normal curvature at $p$. Then for each $\varepsilon>0$ there is a non-critical value $k_{1}$ of $k(s)$ in $\left[k_{0}, k_{0}+\varepsilon\right)$ such that $r^{-1}\left(k_{1}\right)$ contains a non-principal vector $w_{p}$ and hence all the vectors in the connected component of $r^{-1}\left(k_{1}\right)$ which contains $w_{p}$ are non-principal. To find such a non-critical value $k_{1}$ it is sufficient to note that if we consider an open neighbourhood $U$ of $v_{p}$ in $S_{p}(M)=\left\{v \in T_{p} M:|v|=1\right\}$, sufficiently small, then $r(U)$ is of the form $\left[k_{0}, k_{0}+\delta\right), \delta>0$ and $\tau(v) \neq 0$ for each $v \in U-\left\{v_{p}\right\}$.

(2) The point $p$ is umbilical. Let $O$ be the set of umbilical points of $M$. Then there is not an open neighbourhood $U$ of $p$ in $M$ with $U \subset O$. If such an open subset existed, 
then $U$ should be a piece of a plane or of a sphere (Th. 2-2 of [8]). So the value of $r\left(v_{p}\right)=k_{0}$ should be a critical value of $k(s)$ which is absurd. Therefore we can obviously find a sequence $p_{n}$ of non-umbilical points in $M$ converging to $p$. Now using case (1) above we can find a sequence of non-principal vectors $\left(v_{n}\right), v_{n} \in T_{p} M$ such that: the sequence $\left(v_{n}\right)$ converges to $v_{p}$ and the values $r\left(v_{n}\right)=k_{n}$ are non-critical values of $k(s)$ for each $n=1,2, \ldots$. This completes the proof of Lemma 4 .

Now we can finish the proof of the theorem:

The range $R(p)$ of the function $r / S_{p}(M)$ is obviously a closed subset of $R$ and $R(p) \subset R$, for each $p$ in $M$. By Lemmas 3 and 4 at every $p$ in $M$ there are unit tangent vectors $v_{i}$ such that the values $r\left(v_{i}\right)$ form a dense subset in $R$. Therefore $R(p)=\overline{R(p)}=R$. This implies readily that the Gaussian curvature $K$ of $M$ is constant. If $K>0$ then $M$ is compact (Th. 8-18 of [9]) which is impossible. On the other hand a complete surface $M$ of constant negative curvature cannot be embedded in $\mathbb{E}^{3}$ (Th. 5-12 of [8]). Therefore the curvature $K$ of $M$ is equal to zero which implies that $M$ is a generalized cylinder (Th. 5-9 of [8]), and since all geodesics of $M$ are congruent, $M$ will be necessarily an affine plane. But in this case, all geodesics of $M$ are straight lines which contradicts the assumption that the curvature function $k(s)$ is not constant. Therefore $k(s)$ is a constant function and, as explained above, this implies that $M$ is an affine plane.

Remark. In a similar way we can prove the same result for open surfaces $M$ embedded in the hyperbolic space $\mathbb{H}^{3}$. However, since we have not a complete idea for the surfaces of constant curvature in $\mathrm{H}^{3}$ (see $[9$, p. 163]) we proceed as follows: With exactly the same reasonings we conclude that if $\Gamma_{0}$ is not a plane curve and if the curvature of $\Gamma_{0}$ is not constant then the functions of principal curvatures remains constant on $M$. Therefore $M$ is an isoparametric surface in $H^{3}$. The classification of these surfaces which have at most two distinct principal curvatures [1], [7] gives that $M$ is either a geometric sphere or a geometric cylinder or a geodesic plane in $\mathbb{H}^{3}$, and since $\pi_{1}(M)=1$ the result follows.

\section{REFERENCES}

1. E. Cartan, Sur des familles remarquables d'hypersurfaces dans les espaces spheriques, Math. Z. (1939), 335-367.

2. C. Charitos, Surfaces with Congruent Shadow-lines, Mathematika 37 (1990), 43-58.

3. C. Charitos and P. Pamfilos, Surfaces with Isometric Geodesics, Proc. Edinburgh Math. Soc. 34 (1991), 359-362.

4. W. Ballman, E. Ghys, A. Haefliger, P. de la Harpe, E. Salem, R. Strebel, M. Troyanov, Sur les groupes hyperboliques d'après Gromov (Seminaire Berne édité par E. Ghys et $P$. de la Harpe, Birkhauser, 1990).

5. M. HiRsch, Differential Topology (Springer-Verlag, 1976).

6. P. Mani, Fields of planar bodies tangent to spheres, Monatsh. Math. 74 (1970) 145-149.

7. P. RYan, Homogeneity and some curvature conditions for hyperfurfaces, Tôhoku Math. J. 21 (1969), 363-388. 
8. $\left[S_{1}\right],\left[S_{2}\right]$ M. SpIvak, A Comprehensive Introduction to Diff. Geometry. vol III.

9. vol IV (Publish or Perish, 1975).

10. [Su] W. Suss Kennzeichende Eigenschaften der Kugel als Folgerung eines Brouwersche Fixtpunktsatzes, Comment. Math. Helv. 20 (1947), 61-64.

UNIVERSITY OF CRETE

Department of Mathematics

IRAKLION P. O. BOX 1470

GREECE 